\title{
Perfil neuropsicológico de niños con dislalias: alteraciones mnésicas y atencionales
}

\author{
Pablo Conde-Guzón ${ }^{1 *}$, Pilar Quirós-Expósito², María Jesús Conde-Guzón ${ }^{1}$ y María Teresa Bartolomé-Albisteguii ${ }^{3}$ \\ 1 Área de Personalidad, Evaluación y Tratamiento Psicológicos. Universidad de León (León, España) \\ 2 Departamento de Psicología Básica II. UNED. Madrid (España) \\ 3 Servicio de Neurología. Obra Hospitalaria Nuestra Señora de Regla (León, España)
}

\begin{abstract}
Resumen: En este estudio se pretende investigar el funcionamiento neuropsicológico de niños con dislalias. 34 niños con dislalia múltiple (distorsión o sustitución articulatoria de tres fonemas: /r/, / / y /s/) y exploración neurológica normal fueron comparados con 28 niños sin problemas del habla. El perfil neuropsicológico se estima mediante la Escala de Inteligencia Wechsler para Niños-Revisada (WISC-R) y la Batería Neuropsicológica Luria-DNI. Los resultados indican que los niños dislálicos presentan: a) un perfil mnésico globalmente deteriorado respecto al grupo de referencia aunque están preservado la memoria remota y la memoria de formas; b) alteraciones en cinestesia y estereognosia; c) problemas atencionales. Estos resultados indican que los niños dislálicos presentan problemas mnésicos y atencionales similares a los manifestados por niños con problemas de lenguaje secundarios a daño cerebral o afectaciones orgánicas o psicolingüísticas importantes. Debemos considerar este perfil neuropsicológico deteriorado en la orientación neurológica y psicoeducativa de estos niños.

Palabras clave: atención; cinestesia; dislalias; estereognosia; memoria; perfil neuropsicológico.
\end{abstract}

\section{Introducción}

El término dislalia hace referencia a un trastorno primario de la articulación de los fonemas. Es la alteración del habla más frecuente en niños. Se estima que puede tener una incidencia del 5-10\% entre la población infantil. El trabajo pionero de Ingram (1976) ofrece un primer estudio neuropsicológico de los sujetos con problemas primarios de articulación y presenta un modelo con tres niveles en los que ubicar el mecanismo alterado o el tipo de operación deficitaria en cada caso. Así, distingue entre el nivel perceptivo, el organizativo (cognitivo) y el productivo, como posibles núcleos básicos del trastorno. Hasta la publicación de este clásico trabajo en 1976 las dificultades de pronunciación se interpretaban como fallos de aprendizaje del gesto articulatorio (YgualFernández, Cervera-Mérida y Rosso, 2008). Sin embargo, actualmente pensamos que la génesis y el mantenimiento de las dislalias podrían estar sustentados por determinados déficit neuropsicológicos.

A pesar de este primer trabajo de Ingram, el estudio del perfil neuropsicológico completo de los niños con dislalia no despertó un gran interés en la bibliografía revisada.

Todos los estudios se han centrado, por una parte, en la relación entre las dislalias y los problemas de memoria y, por otra, en los problemas articulatorios secundarios a otros cua-

* Dirección para correspondencia [Correspondence address] Pablo A. Conde-Guzón. Área de Personalidad, Evaluación y Tratamiento Psicológicos. Campus de Vegazana. Universidad de León. 24071 León (España). E-mail: pacong@unileon.es
Title: Neuropsychological profile of children with dyslalias: Memory impairment and attentional.

Abstract: In this study we investigate the neuropsychological functioning of children with dyslalias. 34 children with multiple dyslalia (articulatory distortion or substitution of three phonemes: / r /, / 1 / and / s /) and normal neurological examination. 28 children without speech problems are used as reference group. The neuropsychological profile is estimated using the Wechsler Intelligence Scale for Children-Revised (WISC-R) and Luria Neuropsychological Battery-DNI. We found in children with dyslalias: A) The memory profile deteriorated globally in respect to the reference group but are preserved remote memory and the memory of shapes. B) kinesthesia and estereognosia disorders. C) Attentional disorders. We found mnemonic and attentional problems in children with dyslalias similar to those children with language problems secondary to brain damage or affect organs or major psycholinguistic disorders. We consider this neuropsychological profile deteriorated in neurological and psychoeducational counseling, which would justify the treatment and neuropsychological rehabilitation of memory and attention.

Key words: attention; kinesthetic; dyslalias; estereognosia; memory; neuropsychological profile

dros más amplios y en los problemas neuropsicológicos asociados.

Raine y su equipo (Raine, Hulme, Chadderton y Bailey, 1991) son los pioneros en relacionar los trastornos de articulación con una capacidad disminuida en la memoria a corto plazo, una menor longitud de la palabra y una reducida actividad motriz articulatoria. Recientemente se han encontrado problemas prosódicos, alteraciones de memoria auditiva y dificultades en la repetición y en los ritmos auditivos y visuales en sujetos con problemas de articulación (Wells y Peppe, 2003; Baldo y Dronkers, 2006)

También se ha probado que las dificultades de percepción audioverbal, de memoria y de atención, repercuten negativamente en la articulación de la palabra (Klees, 1983).

Por otra parte, existe un gran consenso en recomendar la exploración sistemática de la memoria (mediante la repetición de series de números, palabras y frases) dentro de la evaluación logopédica de niños dislálicos debido a la influencia que los aspectos mnésicos, en general, ejercen sobre el desarrollo del lenguaje oral (Bruno, 1985) y la influencia que la memoria auditiva inmediata, en particular, tiene sobre las dificultades de articulación (Mendoza y Carballo, 1990).

En este mismo sentido, se ha comprobado que la labilidad mnésica dificulta la conceptualización fonológica (Cervera e Ygual, 1994).

El estudio clásico de Baddeley (Gathercole y Baddeley, 1990) postula una relación estrecha entre memoria y velocidad de articulación, siendo la segunda un determinante de la primera. Los niños cuyas capacidades mnésicas son bajas, cometen más errores articulatorios (Adams y Gathercole, 2000). Sin embargo, estos resultados deben tomarse con pre- 
caución, ya que las relaciones entre calidad articulatoria y memoria fonológica son todavía vagas e imprecisas y son necesarios más estudios al respecto. Según algunos autores (Cano y Navarro, 2003), la memoria auditiva (ya sea la memoria a corto plazo, memoria de trabajo y memoria a largo plazo) podría ser uno de los factores que pueden ocasionar un problema fonológico, provocando dificultades en la capacidad de retención o secuenciación de los fonemas.

Los problemas articularios también se han estudiado como síntomas secundarios a alteraciones neurológicas diversas. Así se han encontrado problemas mnésicos, atencionales, visuoconstruccionales y de la función ejecutiva relacionados con dificultades de pronunciación en el marco de los trastornos específicos del lenguaje (Arboleda-Ramírez et al., 2007; Crespo-Eguilaz y Narbona, 2009), en el de las afasias adquiridas y disfasias (Conde-Guzón, Conde-Guzón, Bartolomé-Albistegui y Quirós-Expósito, 2008), en el de las dislexias (Conde-Guzón, Conde-Guzón, Bartolomé-Albistegui, Quirós-Expósito y Cabestrero-Alonso, 2009), en el de los problemas de aprendizaje asociados a problemas cognitivos y comportamentales (Álvarez-Arenal y Conde-Guzón, 2009) o en de niños con epilepsia según el tipo de crisis y farmacología (Conde-Guzón, Bartolomé-Albistegui, Quirós-Expósito y Cabestrero, 2007). Para un estudio detallado sobre los perfiles neuropsicológicos asociados a problemas del lenguaje oral general se puede consultar la revisión de Conde-Guzón y equipo (Conde-Guzón et al., 2009).

Nuestra propia experiencia en el trabajo diario con niños dislálicos nos ha llevado a sospechar la existencia de una cierta dificultad en la capacidad para la memorización y para la concentración. Una buena memoria inmediata auditiva posibilita que el esquema articulatorio se recuerde fácilmente llegando a la automatización. Para comprobar esta hipótesis, Jiménez en 1988 diseñó un estudio con 178 niños de Educación Infantil y $1^{\circ}$ de Primaria y relacionó la memoria inmediata (memoria secuencial auditiva, memoria secuencial de dígitos, memoria de palabras, evocación de objetos y memoria secuencial visual) con los problemas dislálicos. Apreció una alta correlación entre la incapacidad para retener de forma inmediata una información auditiva y la presencia de una dislalia funcional. En consecuencia, en palabras del autor, "se puede confirmar la hipótesis de que las dislalias son frecuentes en aquellos escolares con memoria secuencial auditiva deficiente".

Como se puede ver, la memoria se ha intentado relacionar con problemas de articulación; sin embargo, en el ámbito del idioma castellano no se han realizado estudios del perfil neuropsicológico completo de estos niños. Aparecen algunos estudios que encuentran déficit neuropsicológicos en niños con trastornos articulatorios siempre en el contexto de alteraciones secundarias a otros cuadros como el Trastornos Específicos del Lenguaje (TEL) (Crespo-Eguilaz y Narbona, 2009) o los estudios sobre la rehabilitación neuropsicológica y la relación de la atención, la velocidad del procesamiento, memoria, lenguaje y problemas de la articulación en el marco del daño cerebral adquirido (Noreña et al., 2010).
Para concluir esta revisión, señalamos un estudio reciente (Conde-Guzón, Bartolomé-Albistegui, Quirós y Cabestrero, 2006) que, de manera intencional y sistemática, estudia el funcionamiento mnésico de 150 niños españoles con problemas de articulación de etiología diversa. En esta investigación concluimos que los niños con problemas de articulación del habla presentan dificultades significativas en el funcionamiento mnésico caracterizadas por déficit de memoria remota, memoria visual inmediata, auditiva y lógica, siendo este el primer estudio en el que se encuentran déficit de la memoria visual inmediata en niños con problemas de articulación.

También sospechamos la existencia de alteraciones neuropsicológicas en niños con dislalias primarias. Estos niños se encuentran escolarizados en los centros ordinarios de educación y en raras ocasiones son considerados como grupo de riesgo susceptible de evaluación neuropsicológica y de estudio psicopedagógico. Las administraciones educativas aducen que este grupo no presenta ningún rasgo que justifique su atención especial y, en la mayoría de los casos, pasa desapercibido presentando problemas de enseñanza y aprendizaje a los que no se da la respuesta terapéutica adecuada.

En este contexto, presentamos un estudio del perfil neuropsicológico de los niños con dislalias, sin otros problemas cognitivos y de aprendizaje, escolarizados en centros ordinarios de Educación Primaria. El objetivo es investigar la existencia de ciertos déficits neuropsicológicos asociados a las dislalias. La existencia de dichas alteraciones asociadas, justificaría en sí misma la necesidad de la realización de un diagnóstico y atención neuropsicológicas tempranas cuyo objetivo sería el apoyo especializado en las áreas deficitarias (fundamentalmente en memoria y atención). Este apoyo no suele ser brindado por las administraciones educativas ya que defienden que estos niños no presentan alteraciones importantes que puedan incidir en su rendimiento y en el proceso de enseñanza-aprendizaje.

\section{Método}

\section{Participantes}

La muestra está formada por 34 niños con diagnóstico de dislalia múltiple reclutados de forma correlativa en centros ordinarios de Educación Primaria. Todos los niños poseen un dictamen y diagnóstico de dislalia múltiple (distorsión o sustitución articulatoria de tres fonemas: /r/, /1/ y /s/). Todos presentan una exploración neurológica normal a cargo de un Servicio de Neurología y se ha descartado que presenten TDA (trastorno por déficit de atención). También contamos con un grupo de referencia formado por 28 niños que no manifiestan ningún problema del habla y que está reclutado de forma aleatoria en los mismos centros de enseñanza. Los sujetos de ambos grupos presentan un Cociente de Inteligencia dentro de la normalidad como se describirá más adelante. La proporción de niños/niñas de la muestra es de $6 / 1$ como se puede apreciar en la Tabla 1. 
Tabla 1. Análisis $\chi^{2}$ sobre la frecuencia de antecedentes familiares y sexo entre el grupo con alteraciones del lenguaje y el grupo de referencia.

\begin{tabular}{|c|c|c|c|c|c|}
\hline & \multicolumn{3}{|c|}{$\begin{array}{l}\text { Antecedentes familiares } \\
\left(\chi^{2}=8.09 ; p=.004\right)\end{array}$} & \multicolumn{2}{|c|}{$\begin{array}{c}\text { Sexo } \\
\left(\chi^{2}=10.61 ; p<.001\right)\end{array}$} \\
\hline & $n$ & Sí & No & Masculino & Femenino \\
\hline G. dislalias & 34 & 13 & 21 & 29 & 5 \\
\hline \multirow[t]{3}{*}{ G. referencia } & 28 & 2 & 26 & 13 & 15 \\
\hline & 62 & 15 & 47 & 42 & 20 \\
\hline & & CI Verbal & & lativo & CI Total \\
\hline G. dislalias & & $104.12(D T=13.86)$ & & $=14.06)$ & $104.79(D T=14.52)$ \\
\hline G. referencia & & $107.64(D T=9.16)$ & & $=13.06)$ & $110.04(D T=8.82)$ \\
\hline
\end{tabular}

Estos datos son coherentes con los estudios epidemiológicos que apuntan a la mayor incidencia y prevalencia en varones con patologías debidas a deficiencias de diferentes tipos (Aparicio, 2000: Conde-Guzón et al., 2006; CrespoEguilaz y Narbona, 2009; Conde-Guzón et al., 2009). En nuestro caso es incluso mayor que la presentada en investigaciones sobre dificultades de aprendizaje en general y patologías del lenguaje en particular. De cualquier manera, la mayor prevalencia de trastornos de la articulación en el sexo masculino está bien documentada desde hace varias décadas. Se han descrito gemelos con la misma forma de dislalia. Se han observado caso de dislalia del padre o en la familia paterna más que en la rama materna Adicionalmente se ha constatado que los antecedentes familiares de trastornos lingüísticos influyen en la prevalencia de alteraciones del lenguaje en descendientes. Las familias de niños con trastornos de lenguaje presentan una ratio de 2-3 varones por 1 mujer. Tallal y su equipo (Tallal et al., 1996) han comunicado que en sus muestras hay una proporción más alta de niños afectados que niñas (1.8/1 si el padre estaba afectado, 4/1 si lo estaba la madre y $5 / 1$ si lo estaban ambos progenitores).

El rango de edad en el que se distribuyen los dos grupos escolarizados en los primeros años de Educación Primaria [7 a 9 años; media: 7.6 (0.5)]. No existen diferencias significativas entre la edad del grupo de dislalias y el control. Este nivel de edad permite afirmar el carácter establecido de la alteración del habla como dislalia, edades inferiores podrían justificar el carácter evolutivo de la alteración articulatoria.

Todos los participantes en el estudio tienen una capacidad intelectual normal evaluada con la escala de inteligencia Wechsler de 1993. El CI medio de los grupos es 107.16 (rango: 85-127; desviación típica 12.46).

\section{Instrumentos}

El perfil neuropsicológico se ha obtenido mediante dos instrumentos; WISC-R (Escala de Inteligencia de Wechsler para Niños-Revisada, Wechsler, 1993) y con la Batería Neuropsicológica Luria-DNI (Manga y Ramos, 1991). A continuación se detalla el proceso.

\section{Perfil neuropsicológico WISC-R}

Este perfil se obtiene mediante las puntuaciones en las subáreas del WISC-R. El WISC-R es una prueba ampliamente utilizada en neuropsicología clínica en la edad escolar.
Esto no significa que sea una prueba neuropsicológica, aunque tiene una utilidad importante en el diagnóstico neuropsicológico (Manga y Fournier, 1997). Nyden y su equipo (Nyden, Billstedt, Hjelmquist y Gillberg, 2001) emplean en 2001 los datos obtenidos en el WISC-R para obtener los perfiles neuropsicológicos en niños con déficit de atención con hiperactividad, en Síndrome de Asperger y en niños con trastornos de lectoescritura. A su vez, Otten en 2002 señala que el WISC-R puede ser utilizado con cierta seguridad para el estudio neurolingüístico de sujetos con problemas de comunicación.

\section{Perfil neuropsicológico Batería Luria-DNI Manga y Ramos, 1991)}

Esta batería constituye un instrumento útil y fiable para obtener perfiles neuropsicológicos en niños en el ámbito de la investigación castellano-parlante. Los 19 subtests de la batería Luria-DNI se organizan en 9 pruebas que, a su vez, exploran cuatro amplias áreas de competencias: funciones motoras y sensoriales, lenguaje hablado, lenguaje escrito y aritmética, y memoria. Mediante las tareas propuestas al niño en los 195 ítems de los que está compuesta la prueba, se obtiene el perfil neuropsicológico, que muestra en qué áreas está el niño más fuerte o más débil en comparación con quienes a su misma edad han alcanzado un desarrollo normal de las competencias exploradas. A continuación, en la Tabla 2, puede verse la organización general de la batería Luria-DNI.

\section{Procedimiento}

Previamente a las sesiones de evaluación se solicita el consentimiento informado de los padres de los niños que van a participar en la investigación. Se les explica claramente cuáles son los objetivos, metodología y procedimientos del estudio.

A cada uno de los sujetos se les aplican los dos instrumentos señalados en el apartado anterior (Escala WISC-R y Batería Luria-DNI) para la obtención de los perfiles neuropsicológicos en tres sesiones diferentes:

- Sesión 1: Aplicación de la Escala WISC-R

Para aplicar los 19 subtests de la Batería Luria-DNI es necesario utilizar dos sesiones debido a la extensión de esta prueba. La aplicación se lleva de la siguiente manera: 
- Sesión 2: Aplicación de las pruebas correspondientes a motricidad, audición, tacto-cinestesia, percepción visual, orientación espacial, audición fonémica, comprensión simple y comprensión gramatical.
- Sesión 3: Aplicación de las pruebas correspondientes a análisis fonético, escritura, lectura, estructura numérica, operaciones aritméticas, memoria inmediata y memoria lógica.

Tabla 2. Áreas de la Batería Neuropsicológica Luria-DNI (Manga y Ramos, 1991).

\begin{tabular}{|c|c|c|c|c|}
\hline \multicolumn{2}{|c|}{ Funciones motoras y sensoriales } & Lenguaje hablado & Lenguaje escrito y aritmética & \multirow{2}{*}{$\begin{array}{l}\text { Memoria } \\
\text { Inmediata }\end{array}$} \\
\hline \multirow{2}{*}{ Motricidad } & Manual & Audición fonémica & Análisis fonético & \\
\hline & Regulación verbal & Habla receptiva Comprensión simple & Lectoescritura Escritura & \multirow[t]{2}{*}{ Lógica } \\
\hline \multirow[t]{2}{*}{ Audición } & Estructuras rítmicas & Comprensión gramatical & Lectura & \\
\hline & Tacto & Articulación y repetición & Estructura numérica & \\
\hline Tacto-cinestésia & $\begin{array}{l}\text { Cinestesia y } \\
\text { estereognosia }\end{array}$ & Habla expresiva Denominación y narración & Operaciones aritméticas & \\
\hline \multirow{2}{*}{ Visión } & Percepción visual & & & \\
\hline & Orientación espacial & & & \\
\hline
\end{tabular}

El orden de la aplicación del WISC-R y de la Batería Luria-DNI es contrabandeado con la finalidad de evitar las influencias de las sesiones.

Las sesiones de evaluación son llevadas a cabo de manera individual con todos los sujetos en las primeras horas de la mañana (inicio a las 10 horas) con el fin de minimizar las influencias debidas a la hora del día.

\section{Análisis estadístico}

Todos los análisis estadísticos se realizaron utilizando el software estadístico SPSS v. 19.0 para Windows. Se utilizaron sendos MANOVAs de una vía para analizar las diferencias intergrupos respecto a los perfiles neuropsicológicos evaluados mediante el WISC-R y la Batería Luria-DNI. Con objeto de analizar si los grupos diferían respecto a la variable edad, se utilizó una $t$ de Student para muestras independientes. Para analizar las diferencias en relación a las variables sexo y antecedentes familiares, se empleó el $\chi^{2}$ de Pearson. Como nivel de significación estadística se determinó una $p<.05$.

\section{Resultados}

En primer lugar se analizan las diferencias entre los grupos en relación a las siguientes variables: edad, antecedentes familiares y sexo.

Respecto a la edad, no encontramos diferencias significativas entre los grupos $\left(t_{60}=-1.57 ; M_{\text {dislalias }}=9.02, D T=1.58\right.$; $\left.M_{\text {grefencia }}=9.63, D T=1.48\right)$. Sin embargo, en cuanto a los antecedentes familiares de alteraciones del lenguaje, las diferencias entre los grupos son significativas, presentando el grupo de niños con dislalias una frecuencia mayor de la esperada $\left(\chi^{2}=8.09 ; p=.004 ; \phi=.361\right)$ de antecedentes familiares. Asimismo, los grupos se diferencian también significativamente respecto a la variable sexo, el grupo con dislalias presenta una frecuencia mayor del sexo masculino que la espe- $\operatorname{rada}\left(\chi^{2}=10.61 ; p<.001 ; \phi=.414\right)$. Los datos pueden verse en la tabla 1

\section{Perfiles neuropsicológicos}

Con objeto de comprobar si los perfiles neuropsicológicos, obtenidos mediante el WISC-R y la Batería Luria-DNI, de los niños con dislalias son diferentes de los del grupo sin alteraciones del lenguaje, se realizaron sendos MANOVAs de una vía.

\section{WISC-R}

Los resultados muestran diferencias significativas entre los grupos en relación a la combinación lineal de las distintas subáreas que conforman dicho test $[\mathcal{L}=.677 ; F(11.50)=$ 2.164; $p<.001 ; \eta_{\mathrm{p}}^{2}=.323$ ]. Los posteriores ANOVAs univariados indicaron diferencias significativas entre los grupos en las siguientes pruebas:

- Semejanzas $F(1,60)=4.497 ; p=.038 ; \eta_{\mathrm{p}}^{2}=.07$.

- Dígitos $F(1,60)=4.62 ; p=.035 ; \eta^{2}=.072$.

- Claves $F(1,60)=4.024 ; p=.049 ; \eta_{\mathrm{p}}^{2}=.063$.

Con respecto a semejanzas, el grupo de niños con dislalias presenta puntuaciones significativamente menores que el grupo de referencia; sin embargo, ambos grupos obtienen resultados dentro de la normalidad (por encima del percentil $50)$.

En relación a dígitos y claves, los niños con dislalias presentan puntuaciones por debajo de la media. Esto sugiere un déficit asociado en estos niños en atención concentrada, memoria auditiva inmediata, memoria visual inmediata, previsión asociativa y rapidez motora.

Las puntuaciones obtenidas por los dos grupos en las distintas pruebas del test de WISC-R pueden verse en la figura 1. 


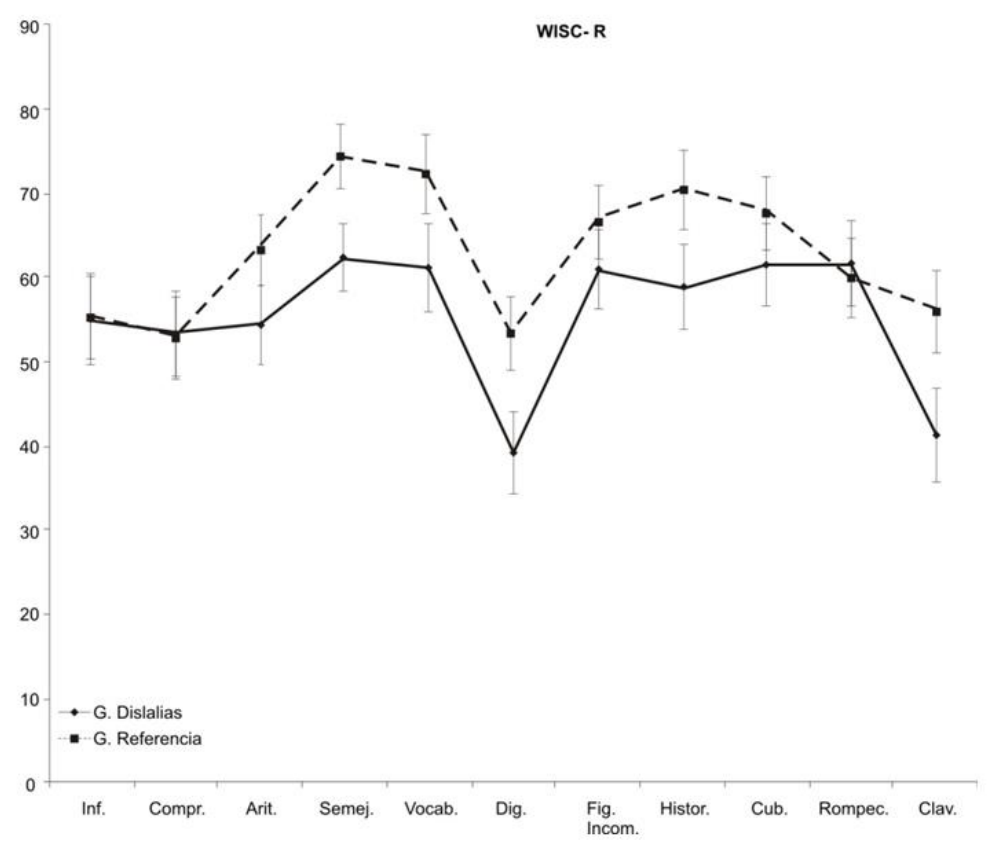

Figura 1. Puntuaciones obtenidas por los dos grupos en el WISC-R. Inf. (Información), Compr. (Comprensión), Arit. (Aritmética), Semej. (Semejanzas), Vocab. (Vocabulario), Dig. (Dígitos), Fig. Incom. (Figuras Incompletas), Histor. (Historietas), Cub. (Cubos), Rompec. (Rompecabezas), Clav. (Claves).

Teniendo en cuenta las puntuaciones obtenidas en el CI verbal y en el CI manipulativo, no se encuentran diferencias significativas entre los grupos $[\mathcal{L}=.953 ; F(2,59)=1.442 ; p=$ .245]. Los CIs obtenidos por ambos grupos se pueden ver en la Tabla 1.

\section{Batería Luria-DNI}

Los resultados del MANOVA muestran diferencias significativas entre los grupos en relación a la combinación lineal de los 19 subtest que componen la batería $[\mathcal{Z}=.155$; $\left.F(19,42)=12.079 ; p<.001 ; \eta_{\mathrm{p}}^{2}=.845\right]$. Los posteriores ANOVAs univariados indican diferencias significativas entre los grupos en todos los subtest, exceptuando comprensión simple y comprensión gramatical. Los resultados de estos ANOVAs se exponen en la Tabla 3.

Las puntuaciones obtenidas por los dos grupos en la batería LURIA-DNI pueden observarse en la Figura 2.

El grupo de referencia presenta, en todos los subtest, puntuaciones superiores al grupo de niños dislálicos. Sin embargo, este último grupo obtiene, en la mayoría de las pruebas, puntuaciones que se sitúan dentro de la zona considerada normal (entre los percentiles 40 y 60). Las puntuaciones que se sitúan por debajo de esta zona corresponden, en su mayor parte, a subtest relacionados con los problemas específicos de este grupo (audición fonémica, articulación y repetición, denominación y narración, escritura y lectura, co- rrespondiendo la puntuación más baja — como era de esperar- a articulación y repetición). Es de destacar, no obstante, que los niños dislálicos han puntuado en esta batería por debajo de la zona de puntuaciones considerada normal en las dos pruebas que miden memoria y en cinestesia y estereognosia.

Tabla 3. Diferencias significativas entre los grupos de dislálicos y referencia (Batería Neuropsicológica Luria-DNI.

\begin{tabular}{llll}
\hline & $F(1,60)$ & $p$ & $\eta^{2} \mathrm{p}$ \\
\hline Manual (Ma) & 41.186 & $<.001$ & .407 \\
Regulación verbal (R.V.) & 11.025 & $=.002$ & .155 \\
Estructuras rítmicas(E.R.) & 8.716 & $=.004$ & .127 \\
Tacto (Ta) & 13.772 & $<.001$ & .187 \\
Cinestesia y esterognosia (C.E.) & 9.646 & $=.003$ & .139 \\
Percepción visual (P.V.) & 12.711 & $=.001$ & .175 \\
Orientación espacial (O.E.) & 14.434 & $<.001$ & .194 \\
Audición fonémica (A.F.) & 15.014 & $<.001$ & .200 \\
Articulación y repetición (A.R.) & 106.507 & $<.001$ & .640 \\
Denominación y narración (D.N.) & 24.852 & $<.001$ & .293 \\
Análisis fonético (A.F.) & 17.415 & $<.001$ & .225 \\
Escritura (Esc) & 7.295 & $=.009$ & .108 \\
Lectura (Lec) & 13.258 & $=.001$ & .181 \\
Estructura numérica (E.N.) & 10.768 & $=.002$ & .152 \\
Operaciones aritméticas (O.A.) & 5.014 & $=.029$ & .077 \\
Memoria inmediata (M.I.) & 14.877 & $<.001$ & .199 \\
Memoria lógica (M.L.) & 18.487 & $<.001$ & .236 \\
\hline
\end{tabular}




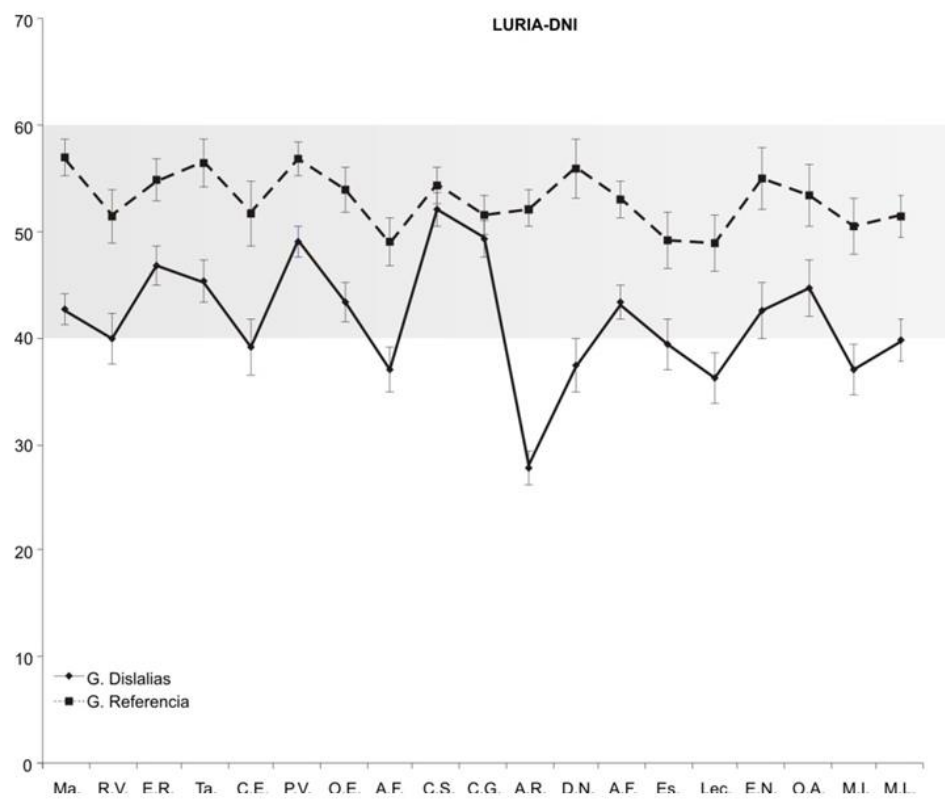

Figura 2. Puntuaciones obtenidas por los dos grupos en la Batería Luria-DNI

Manual (Ma), Regulación verbal (R.V.), Estructuras rítmicas(E.R.), Tacto (Ta), Cinestesia y esterognosia (C.E.), Percepción visual (P.V.), Orientación espacial (O.E.), Audición fonémica (A.F.), Articulación y repetición (A.R.), Denominación y narración (D.N.), Análisis fonético (A.F.), Escritura (Esc), Lectura (Lec), Estructura numérica (E.N.), Operaciones aritméticas (O.A.), Memoria inmediata (M.I.), Memoria lógica (M.L.)

Teniendo en cuenta los resultados de estas dos pruebas, y considerando, además, los subtest que evalúan la memoria en el test de WISC-R, podemos obtener un perfil mnésico formado por los siguientes subtest: Información (memoria remota), Dígitos (memoria auditiva inmediata), Figuras In- completas (memoria visual), Rompecabezas (memoria de formas), Claves (memoria visual inmediata), Memoria Inmediata y Memoria Lógica. El perfil mnésico se muestra en la Figura 3.

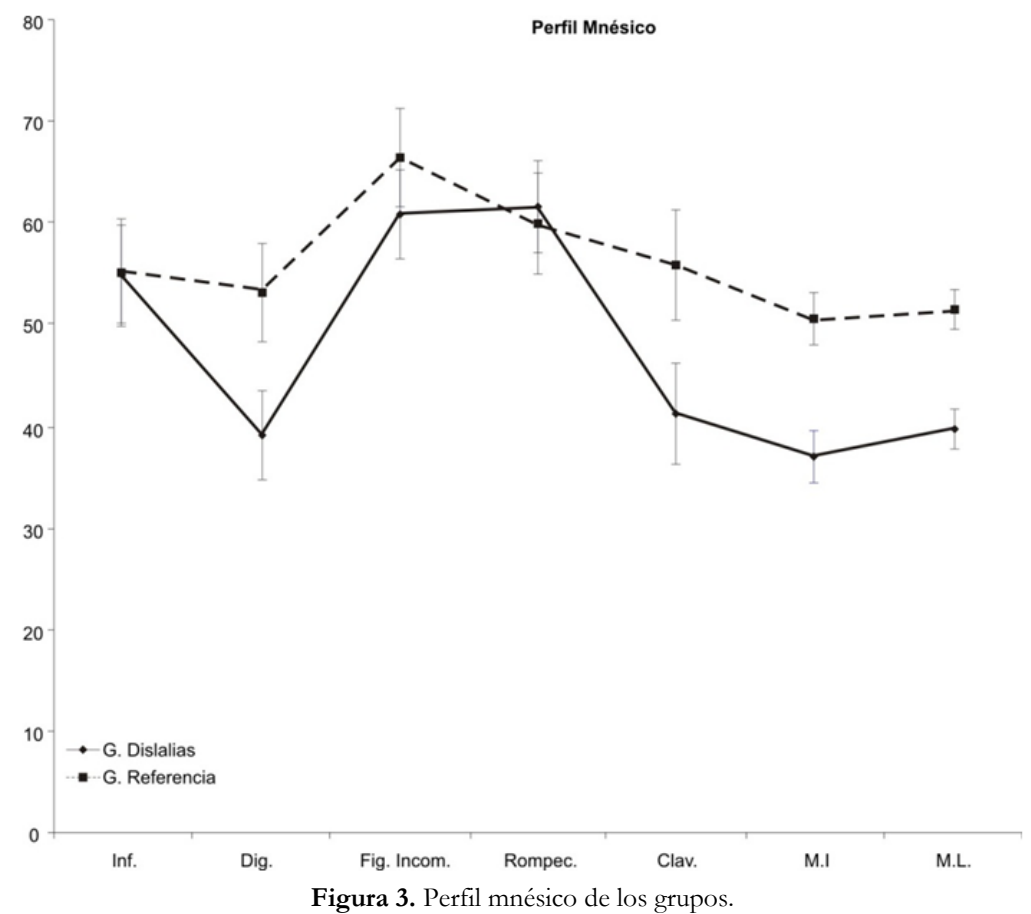


Como se puede observar en el gráfico, los niños dislálicos presentan un perfil mnésico globalmente deteriorado (excepto para la memoria remota y la memoria de formas).

Por otra parte, como se ha mencionado con anterioridad, estos niños podrían manifestar asimismo problemas atencionales. Si bien la única prueba con significación neuropsicológica para la estimación directa es el subtest de Dígitos del WISC-R (mide la atención concentrada —además de la me- moria auditiva inmediata-), podemos utilizar el "tercer factor" del WISC que es un índice cognitivo complejo asociado con la capacidad de enfocar y mantener la atención mientras se realizan tareas cognitivas superiores. Este perfil atencional se obtiene con las puntuaciones en los subtest de Aritmética, Dígitos, Claves. Las puntuaciones obtenidas por los dos grupos se presentan en la Figura 4.

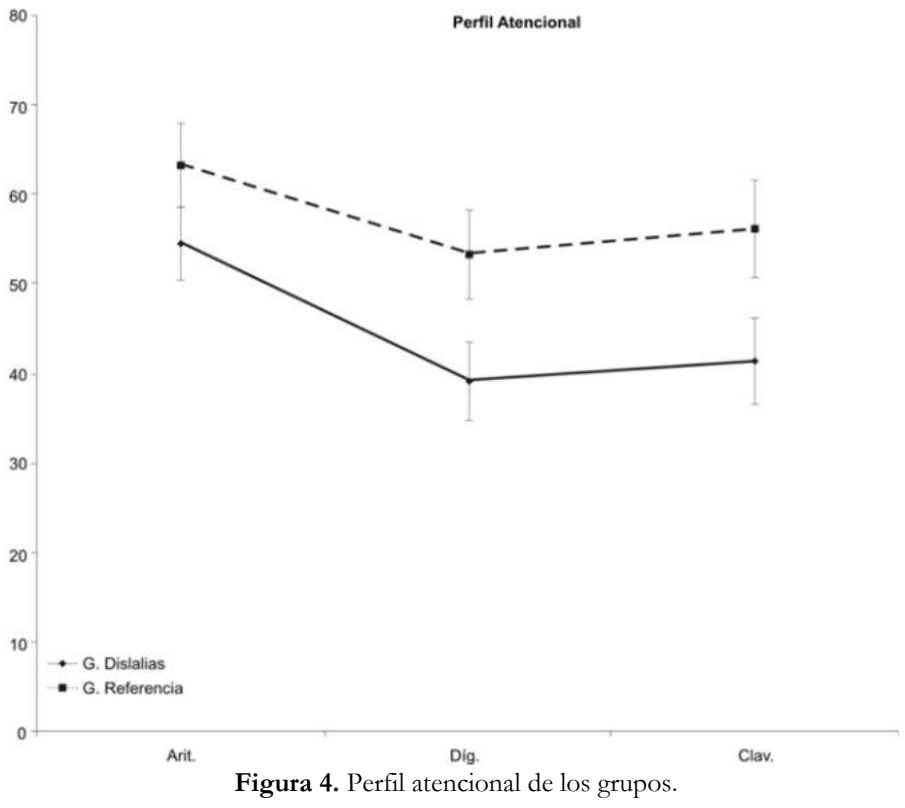

Como se puede observar, en estos tres subtest, los niños con dislalias presentan puntuaciones inferiores respecto al grupo de referencia. Siendo significativas las diferencias entre ambos grupos en Dígitos y Claves.

\section{Discusión}

De los resultados obtenidos podemos concluir que los niños con dislalias manifiestan problemas neuropsicológicos; básicamente en déficit mnésicos (fundamentalmente de memoria inmediata), problemas atencionales y alteraciones de la cinestesia y estereognosia. Además, como es obvio, manifiestan problemas severos en análisis fonético, articulación, narración, repetición, escritura y lectura.

1.- Hemos encontrado en los niños con dislalias una mayor frecuencia de antecedentes familiares de problemas articulatorios que en los niños del grupo de referencia. $\mathrm{Na}$ die pone en duda, actualmente, la alta incidencia de trastornos del lenguaje en general entre niños con antecedentes familiares de alteraciones de lenguaje. En este sentido, aparte de las evidencias sobre la predisposición genética a sufrir patologías del lenguaje que se describen en los manuales de trastornos de lenguaje, merece la pena consultar la revisión sobre los factores de riesgo debidos a la herencia, que realizan Barry y colaboradores (Barry, Ya- sin y Bishop, 2007). Estos autores confirman la mayor prevalencia de problemas de lenguaje en hijos de padres con patología lingüísticas $(32 \%)$ cuando se comparan con padres no afectados (6\%). Mulas y equipo (Mulas, Morant, Rosselló, Soriano e Ygual, 1998) revisan la literatura dirigida a clarificar la posible asociación entre factores de riesgo y dificultades de aprendizaje en general, y concluyen que en los problemas de lenguaje, principalmente en la dislexia, existe una alta carga genética. Por otra parte, también se ha demostrado la alta heredabilidad en la competencia fonológica. En casos excepcionales se han descrito alteraciones en la capacidad del lenguaje ligadas a mutaciones únicas de un gen (FOXP2). El descubrimiento de la mutación del gen FOXP2 en una familia inglesa (KE) ha abierto una apasionante vía para el conocimiento de los mecanismos del aprendizaje del habla. No obstante, desde el punto de vista genético, hay que recordar que el lenguaje es un fenómeno muy complejo de modo que, en la gran mayoría de los casos, los trastornos específicos del lenguaje obedecen a la interacción de múltiples genes (Sanjuán et al., 2010). Un alto porcentaje de concordancia para trastornos de lenguaje se observó en mellizos monocigotos, frecuentemente con similar patrón de alteración (Bishop, North y Donlan, 1995; Tomblin y Buckwalter, 1998; Castaño, 2003). Por otra parte, se ha 
observado mayor incidencia de trastornos del lenguaje en niños con hiperplasia adrenal congénita, hallazgo que ha generado la sospecha de que existe una asociación con el gen para este trastorno (Plante, Boliek, Binkiewicz y Erly, 1996; Castaño, 2003). La investigación sobre el cromosoma $7 q$ y su posible relación con los trastornos del lenguaje ha mostrado algunos resultados positivos, aunque en escala limitada (Warburton et al., 2000; Castaño, 2003).

2.- Los niños con dislalias de nuestro estudio presentan un perfil manipulativo preservado como el observado en los estudios previos de Conde-Guzón y equipo (CondeGuzón et al., 2006; 2009). Sin embargo, además de los problemas de memoria auditiva inmediata, encontramos un deterioro de la memoria visual inmediata y la rapidez motora estimadas con la prueba de claves (WISC-R) en niños dislálicos sin otros problemas asociados. Encontramos un deterioro mnésico similar al de otros estudios previos de nuestro grupo con niños con problemas secundarios a alteraciones cerebrales evidentes como en las afasias y disartrias (Conde-Guzón et al., 2008) o en niños con epilepsia (Conde-Guzón, et al., 2007).

Es éste un hecho novedoso, ya que el deterioro en la memoria visual inmediata y el deterioro de memoria general y verbal no se han relacionado con dislalias primarias. Con respecto al deterioro de la memoria visual inmediata, se ha encontrado un déficit de la memoria visual inmediata en niños con dislalias asociadas a problemas de lectoescritura (Estévez y García, 1996), al subtipo perceptivo-visual de niños disléxicos (Manga y Fournier, 1997), a discalculia (Swanson y Sache-Lee, 2001) y a las disgrafías de tipo fonológico (Dodd, 1993). Con respecto al deterioro en memoria verbal y general, se han encontrado en el contexto de los problemas articulatorios secundarios a otros cuadros. Así, tras el estudio pionero de Ingram en 1976 y del estudio clásico de Baddeley y su equipo en 1974 (Baddeley y Hitch, 1974) que postulaba una estrecha relación entre la memoria y velocidad de articulación, hay que esperar a la década de los 90 para que aparezcan estudios que relacionen los trastornos fonológicos generales con problemas de memoria verbal en niños con problemas de articulación siempre secundarios a otras patologías (Raine, Hulme, Chadderton y Bailey, 1991; Kiese-Himmel y Kruse, 1998; Baldo y Dronkers, 2006; Ortiz-Siordia, Álvarez-Amador y González-Piña, 2008). En este contexto, Adams y Gathercole en el 2000 demuestran que los niños cuyas capacidades mnésicas son bajas, cometen más errores articulatorios que los niños con mejores capacidades. Sin embargo, estos resultados deben tomarse con precaución, ya que el número de errores articulatorios producidos por los grupos no era significativamente distinto. De cualquier manera, parece ser que la memoria de trabajo, más específicamente el bucle fonológico, contribuye al desempeño en las tareas de fluidez verbal fonológica (Arán-Filippetti, 2011). Las relaciones entre la calidad articulatoria y memoria fono- lógica son aun vagas e imprecisas y son necesarios más estudios al respecto. El estudio de Jiménez que hemos señalado en la introducción (Jiménez, 1988) es el único que intenta relacionar las dificultades mnésicas en niños con dislalias. En él se encuentra una deficiente memoria secuencial auditiva en los niños con problemas articulatorios.

3.- Por otra parte, el hallazgo de problemas de rapidez motora en estos niños también es novedoso. Estudios recientes han encontrado problemas de rapidez motora y velocidad en secuencias motrices finas en niños con afasias y disfasias (Tallal et al., 1996) y en niños con problemas de lectoescritura (Baldo y Dronkers, 2006); pero no hemos encontrado estudios que relacionen estos problemas con niños dislálicos.

4.- La alteración en cinestesia y estereognosia en los niños con dislalias es un hecho que tampoco aparece en la literatura revisada. Aunque se encuentran dificultades cinestésicas y estereognósicas en niños afásicos, disártricos, o incluso disgráficos, solamente se ha encontrado un estudio que desvela una correlación significativa entre los problemas de lenguaje oral y la actuación táctilcinestésica en niños con CI manipulativo similar (KieseHimmel y Kruse, 1998). Esta relación encontrada podría apoyar la implicación de las estructuras parietales en el origen de los problemas de articulación. Desde esta óptica, no solamente estructuras de control motriz estarían modulando el habla sino que se reforzaría la coexistencia de un control sensitivo que sustentaría las dislalias de etiología discriminativa (fonológica). De cualquier manera, los circuitos de interconexión cerebral, en este ámbito, son complicados y desconocidos desde el punto de vista funcional.

5.- Las alteraciones en repetición podrían sugerir disfunciones premotoras que, si bien no causan dificultades primarias de articulación, producen déficit en el habla repetitiva como actividad eminentemente secuencial (Manga y Ramos, 1991). Aunque también podríamos hipotetizar que estas disfunciones podrían estar relacionadas con alteraciones de otras zonas del sistema nervioso central que están implicadas en las dificultades en la repetición de las sílabas, ya que como señala el equipo de Ortiz (Ortiz-Siordia, Álvarez-Amador y González-Piña, 2008), las áreas que se activan durante la repetición de sílabas son el giro supratemporal, la región precentral de la corteza motora y premotora izquierda, el putamen y parte del cerebelo. Como vemos se trata de complejas conexiones funcionales.

6.- Otro resultado relevante en nuestro estudio es el hallazgo de déficit atencional en los niños con problemas articulatorios primarios. Los problemas atencionales han sido frecuentemente observados en niños con alteraciones lingüísticas generales fundamentalmente en el marco del Trastorno Específico del Lenguaje (TEL). En este sentido, el equipo de Buiza-Navarrete (Buiza-Navarrete, Adrían-Torres y González-Sánchez, 2007) ha encontrado 
limitaciones del TEL en atención, codificación, memoria y función ejecutiva y confirman estudios previos realizados con niños de otros idiomas. En palabras de los autores, los resultados obtenidos en este trabajo aportan algunas estrategias para su evaluación diagnóstica aunque se extienden a un campo más amplio de dificultades en las que las dislalias son solamente un síntoma dentro de síndromes más generales.

Los problemas atencionales podrían estar implicados en la génesis y desarrollo de las dislalias ya que como explican Mulas y equipo (Mulas, Etchepareborda, Diaz-Lucero y Ruiz-Andrés, 2006), las dificultades en los aspectos fonéticofonológico y gramaticales están probablemente causadas por una dificultad de atención y déficit en el control inhibitorio de estímulos irrelevantes, más que por una incapacidad específica para manejar estos aspectos lingüísticos.

En este sentido, aunque es muy arriesgado, nosotros pensamos que estos niños se podrían beneficiar de los efectos del metilfenidato que tan buenos resultados ha dado en niños con TDAH bien diagnosticado. Al mejorar este fármaco directamente la atención e indirectamente la memoria, podría repercutir positivamente sobre las alteraciones de la articulación. Si bien algunos autores como Miranda et al. (Miranda-Casas, Ygual-Fernández, Mulas-Delgado, RosellóMiranda y Bó, 2002) o, incluso hace bastantes años, el equipo de Malone (Malone, Kerschner y Siegel, 1988) han sugerido la posibilidad de que la medicación mejore específicamente el procesamiento fonológico de escolares de Educación Primaria, los análisis de la eficacia del mentilfenidato en este contexto no son concluyentes debido probablemente a que la medicación ejerce un efecto facilitador en aspectos generales del procesamiento cognitivo y no en aspectos más específicos como la articulación. No obstante, sería intere-

\section{Referencias}

Adams, A. y Gathercole, S. (2000). Limitations in working memory: implications for language development. International Journal of Language and Communication Disorders, 35(1), 95-116

Alvarez-Arenal, T. y Conde-Guzón, P.A. (2009). Formación de subtipos de niños con problemas escolares de aprendizaje a partir de la evaluación neuropsicológica, capacidades cognitivas y comportamentales. Clínica y salud, 20(1), 19-43.

Aparicio, J. M. (2000). Neurología Pediátrica. Madrid: Ergon.

Arán-Filippeti, V. (2011). Fluidez verbal según el tipo de tarea, intervalo de tiempo y estrato socioeconómico en niños escolarizados. Anales de Psicologia, 27(3), 816-826.

Arboleda-Ramírez, A., Lopera-Vásquez, J.P., Hincapié-Henao, L., GiraldoPrieto, M., Pineda, D.A., Lopera, F. et al. (2007). Trastorno específico del desarrollo del lenguaje: problema selectivo o generalizado de la cognición. Revista de Neurologia, 44, 596-600.

Baddeley, A. y Hitch, G. (1974). Working memory. En G. H. Bower (Ed.), The psychology of learning and motivation: Advances in research and theory (Vol. 8, pp. 47-90). San Diego, CA: Academic Press.

Baldo, J. y Dronkers, N (2006). The role of inferior parietal and inferior frontal cortex in working memory. Neuropsychology, 20, 529-38.

Barry, J., Yasin, I. y Bishop, D. (2007). Heritable risk factors associated with language impairments. Genes, Brain and Behavior, 6 (1), 66-76. sante ensayar los beneficios del metilfenidato en niños dislálicos. Asimismo, sería conveniente comprobar el funcionamiento específico de la Memoria de Trabajo de estos niños debido a la interrelación que existe entre este tipo de memoria y la atención. Esta relación se centra en que la Memoria de Trabajo engloba los contenidos de la Memoria a Corto Plazo que se encuentran bajo control atencional (GarcíaMolina, Tirapu-Ustárroz, Luna-Lario, Ibáñez y Duque, 2010).

En conclusión, y a pesar de recomendar nuevos estudios aumentando el tamaño de la muestra y comprobando el funcionamiento mnésico y atencional con otro tipo de pruebas más específicas, podemos afirmar que el hallazgo de los déficit mnésicos y atencionales justifica en sí mismo, la necesidad de realizar un diagnóstico precoz del perfil neuropsicológico de estos niños con la finalidad de realizar una atención directa (apoyo neuropsicológico y psicopedagógico) en el ámbito de integración de los centros de escolarización. Esta necesidad debe ser asumida por las administraciones educativas que hasta ahora consideran las dislalias como problemas menores sin afectación de otras áreas. Los problemas mnésicos en niños con dislalias encontrados son similares a los que manifiestan niños con problemas de lenguaje secundarios a daño cerebral o afectaciones orgánicas importantes. Debemos considerar este perfil neuropsicológico deteriorado en la orientación neurológica y psicoeducativa de estos niños. Este perfil justifica el tratamiento neuropsicológico y la rehabilitación de la memoria y la atención. Asimismo, pensamos que estos niños podrían beneficiarse de los efectos positivos del metilfenidato que viene siendo utilizado con resultados positivos sobre los aspectos cognitivos y conductuales en niños con TDAH (con los que existe alta comorbilidad).

Bishop, D. V., North, T. y Donlan, C. (1995). Genetic basis of specific language impairment: evidence from a twin study. Developmental Medicine and Child Neurology, 37, 56-71.

Bruno C. (1985). Cómo abordar la exploración logopédica en el niño. Revista de logopedia, foniatría y audiología, 5(2), 69-87.

Buiza-Navarrete, J., Adrían-Torres, J. y González-Sánchez, M. (2007). Marcadores neurocognitivos en el trastorno específico del lenguaje. Revista de Neurología, 44, 326-33.

Cano, M. y Navarro, M. (2003). Dificultades en el desarrollo del habla y del lenguaje oral en la infancia y la adolescencia. En M. Puyuelo y J. A. Rondal (Eds.), Manual de desarrollo y alteraciones del lenguaje (pp. 323-368). Barcelona: Masson.

Castaño, J. (1994). Bases neurobiológicas del lenguaje y sus alteraciones. Revista de Neurología, 36(8), 781-785.

Cervera, J. e Ygual, A. (1994). Metodología para la intervención logopédica en trastornos del habla. Revista de logopedia, foniatría y audiología, 14(1), 1927.

Conde-Guzón, P. A., Bartolomé-Albistegui, M. T., Quirós, P. y Cabestrero, R. (2006). Deterioro mnésico en niños con problemas de lenguaje oral. Neurología, 21(9), 608.

Conde-Guzón, P. A., Bartolomé-Albistegui, M. T., Quirós-Expósito, P. y Cabestrero, R. (2007). Perfiles neuropsicológicos en niños con epilepsia según el tipo de crisis y farmacología. Neurología 2007, 22(9), 688-689. 
Conde-Guzón, P. A., Conde-Guzón, M. J., Bartolomé-Albistegui, M. T. y Quirós-Expósito, P. (2008). Perfil neuropsicológico en niños con afasias adquiridas y disfasias. Neurología, 23(10), 790.

Conde-Guzón, P. A., Conde-Guzón, M.J., Bartolomé-Albistegui, M. T., Quirós-Expósito, P. y Cabestrero-Alonso, R. (2009). Perfil neuropsicológico de niños con dislexia ¿Implicaciones farmacológicas? Neurología, 24,778 .

Conde-Guzón, P. A., Conde-Guzón, M. J., Bartolomé-Albistegui, M. T. y Quirós-Expósito, P. (2009). Perfiles neuropsicológicos asociados a problemas del lenguaje oral infantil. Revista de Neurología, 48(1), 32-38.

Crespo-Eguilaz, N. y Narbona, J. (2009). Trastorno de aprendizaje procedimental: Características neuropsicológicas. Revista de Neurología, 49(8), 409-416.

Dodd, B. (1993). Speech disordered children. En G. Blanken (Ed.). Linguistic disorders and pabtologies: An internacional handbook. Berlin: De Gruyter.

Estévez, A. y García, C. (1996). La dislexia. Estado actual de nuestros conocimientos neurológicos y neuropsicológicos. Revista de Neurología, 24(125), 31-39.

García-Molina, A., Tirapu-Ustárroz, J., Luna-Lario, P., Ibáñez, J. y Duque, P. (2010). ¿Son lo mismo inteligencia y funciones ejecutivas?. Revista de Neurología, 50(12), 738-746.

Gathercole, S. E. y Baddeley, A. D. (1990). Phonological memory deficits in language disordered children: Is there a causal connection? Journal of Memory and Language, 29, 336-360.

Ingram, D. (1976). Phonological disability in children. Londres: Edward Arnold.

Jiménez, J. (1988). Imbricación de la memoria en la génesis de la dislalia funcional. Revista de logopedia, foniatría y audiología, 8(3), 173-182.

Kiese-Himmel, C. y Kruse, E. (1998). Higher touch and kinesthetic functions in children with former speech/language development disorders: a neuropsychological study. Folia Phoniatrica et Logopaedica, 50(4), 195204

Klees, M. (1983). A propósito de los trastornos instrumentales asociados a trastornos de aprendizaje precoces de lectura, ortografía y cálculo en el uso inteligente: la noción de gravedad. Revista de logopedia, foniatría y audiología, 11(3), 139-153.

Malone, M. A., Kerschner, J. R. y Siegel, L. (1988). The effects of metilphenidate on levels of processing and laterality in children with attention deficit disorder. Journal of Abnormal Child Psychology, 16, 379-95.

Manga. D., y Fournier, C. Neuropsicología clínica infantil. Estudio de casos en edad escolar. Madrid: Universitas; 1997.

Manga, D. y Ramos, F. (1991). Neuropsicología de la edad escolar. Aplicaciones de la teoría de A.R. Luria a niños a través de la Batería Luria-DNI. Madrid: Visor.

Mendoza, E. y Carballo, G. (1990). La evaluación del lenguaje en edad preescolar. Revista de logopedia, foniatría y audiología, 10(2), 84-92.

Miranda-Casas, A., Ygual-Fernández, A., Mulas-Delgado, F., RosellóMiranda, B. y Bó, R. (2002). Procesamiento fonológico en niños con trastorno por déficit de atención con hiperactividad: ¿es eficaz el metilfenidato?. Revista de Neurología, 34(1), S115-S121.

Mulas, F., Etchepareborda, M., Diaz-Lucero, A. y Ruiz-Andrés, R (2006). El lenguaje y los trastornos del neurodesarrollo. Revista de Neurología, 42(2), S103-S109.
Mulas, M., Morant, A., Rosselló, B., Soriano, M. e Ygual, A. (1998). Factores de riesgo de las dificultades en el aprendizaje. Revista de Neurología, 27, 274-279.

Noreña, D., Rios-Lago, M., Bombín-González, I., Sánchez-Cubillo, I., García-Molina, A. y Tirapu-Ustárroz, J. (2010). Efectividad de la rehabilitación neuropsicológica en el daño cerebral adquirido (I): atención, velocidad de procesamiento, memoria y lenguaje. Revista de Neurología, 51(11), 687-698.

Nyden, A., Billstedt, E., Hjelmquist, E. y Gillberg, C. (2001). Neurocognitive stability in Asperger syndrome, ADHD, and reading and writing disorder: a pilot study. Developmental Medicine and Child Neurology, 43, 16571.

Ortiz-Siordia, L., Álvarez-Amador, L. y González-Piña, R. (2008). Modelos anatomopatológicos de las áreas cerebrales que se activan durante la función lingüística. Revista de Neurología, 47(12), 653-658.

Ottem, E. (2002). Do the Wechsler scales underestimate the difference between verbal and performance abilities in children with language-related disorders? Scandinavian Journal of Psychology, 43, 291-298.

Plante, E., Boliek, C., Binkiewicz, A. y Erly, W. K. (1996). Elevated androgen, brain development and language/learning disabilities in children with congenital adrenal hyperplasia. Developmental Medicine and Child Neurology, 38, 423-37.

Raine, A., Hulme, C., Chadderton, H. y Bailey, P. (1991). Verbal short-term memory span in speech-disordered children: implications for articulatory coding in short-term memory. Child Develpment, 62(2), 415-423.

Sanjuán, J., Tolosa, A., Colomer-Revuelta, J., Ivorra-Martínez, J., Llacer, B. y Jover, M. (2010). Factores genéticos en el desarrollo del lenguaje. Revista de Neurología, 50(3), S101-S106.

Swanson, H. y Sache-Lee. (2001). Mathematical problem solving and working memory in children with learning disabilities: both executive and phonological processes are important. Journal of Experimental Child Psychology, 79, 294-321.

Tallal, P., Miller, S., Bedi, G., Byma, G., Wang, X. et al. (1996). Language comprensión in language-learning imparied children improved with acoustically modified speech. Science, 271, 81-84.

Tomblin, J. B. y Buckwalter, P. R. (1998). Heritability of poor language achievement among twins. Journal of Speech, Language and Hearing Research, $41,188-99$

Warburton, P., Baird, G., Chen, W., Morris, K., Jacobs, B. W., Hodgson, S. et al. (2000). Support for linkage of autism and specific language impairment to $7 \mathrm{q} 3$ from two chromosome rearrangements involving band 7q31. American Journal of Medicical Genetics, 96, 228-34. d

Wechsler D. (1993). Escala de inteligencia Wechsler para niños, revisada (WISC-R). Madrid: TEA Ediciones.

Wells, B. y Peppe, S. (2003). Intonation abilities of children with speech and language impairments. Journal of Speech, Language and Hearing Research, 46(1), 5-20.

Ygual-Fernández, A., Cervera-Mérida, J. F. y Rosso, P. (2008). Utilidad del análisis fonológico en la terapia del lenguaje. Revista de Neurología, 46(1), S97-S100.

(Articulo recibido: 17-4-2012; revisión revisada: 27-11-2013; aceptado: 20-3-2014) 